\title{
Importance of the formulation in the skin delivery of topical diclofenac: not all topical diclofenac formulations are the same
}

Julie Pradal

Coralie M Vallet

Guillaume Frappin

Frédérique Bariguian

Maria Stella Lombardi

Department of New Product Development, R\&D, GSK Consumer Healthcare S.A, Nyon I, 1260,

Switzerland
This article was published in the following Dove Press journal: Journal of Pain Research

Purpose: The current study aimed to compare 2 topical diclofenac products (diclofenac diethylamine [DEA] $1.16 \%$ emulsion and diclofenac sodium [Na] $5 \%$ gel). The quantitative evaluation of skin permeability and the qualitative evaluation of their physical characteristics were performed.

Methods: The skin permeability of diclofenac DEA $1.16 \%$ emulsion and diclofenac Na $5 \%$ gel was compared in vitro using Franz diffusion cells following a single, fixed, $10 \mathrm{mg} / \mathrm{cm}^{2}$ dose of product applied to a $0.64 \mathrm{~cm}^{2}$ area of the stratum corneum surface of ex vivo human skin samples. The physical characteristics of the 2 formulations were assessed by rheological measurement and microscopy observation.

Results: Diclofenac DEA $1.16 \%$ emulsion exhibited a statistically significant higher permeation through human skin at $24 \mathrm{hrs}$ than diclofenac Na 5\% gel $\left(554\right.$ vs $361 \mathrm{ng} / \mathrm{cm}^{2}$, respectively; ratio of adjusted geometric means, 1.54 [95\% CI, 1.14-2.07]). When expressed as a percentage of the applied dose of diclofenac that permeated through human skin, a 7 -fold difference was observed between the diclofenac DEA $1.16 \%$ emulsion $(0.54 \%)$ and the diclofenac Na 5\% gel (0.077\%). Qualitative composition and physical characterization showed differences between the formulations that may explain some of the permeation data observed. Based on rheological assessments, diclofenac $\mathrm{Na} 5 \%$ gel had a higher viscosity (24.82 Pa.s) than diclofenac DEA $1.16 \%$ emulsion (10.29 Pa.s).

Conclusion: A topical diclofenac product with a higher concentration of the active ingredient does not necessarily lead to greater absorption relative to a product with lower concentration of the active ingredient but different characteristics. These observations highlight the importance of considering parameters beyond drug concentration, such as composition, which may influence the solubility of the drug and permeation of topical nonsteroidal anti-inflammatory drugs.

Keywords: topical application, excipients, nonsteroidal anti-inflammatory drug, physicochemical properties, Voltaren

\section{Introduction}

Nonsteroidal anti-inflammatory drugs (NSAIDs) are commonly used to treat acute and chronic pain, ${ }^{1,2}$ but their long-term use may be limited by systemic side effects such as gastrointestinal toxicity and the potential for certain cardiovascular and

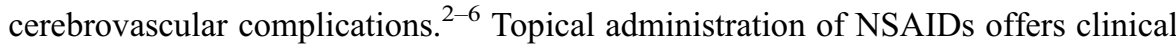
efficacy similar to oral NSAIDs, fewer systemic adverse events, and a reduced risk of drug-drug interactions. ${ }^{7-10}$ The NSAID diclofenac has demonstrated efficacy in
Correspondence: Julie Pradal Department of New Product Development, R\&D,GSK Consumer Healthcare S.A.,

Route de l'Etraz 2, 1260 Nyon I,

Switzerland

Tel +4 I22 5672672

Email Julie.x.pradal@gsk.com 
treating a variety of acute and chronic pain conditions. ${ }^{2,11}$ Diclofenac is a good choice of topical NSAID based on a number-needed-to-treat of 1.8 for acute pain ${ }^{12}$ and 9.8 for chronic pain, ${ }^{13}$ as reported in a recent Cochrane meta-analysis. Topical diclofenac is available in several different forms, including gel, spray, emulsion, aqueous solution, cream, and transdermal patch. ${ }^{2,10}$

Although it may be assumed that the skin permeation will be directly proportional to the drug concentration, according to Fick's law, other drug physicochemical parameters may influence drug permeation. According to O'Connor et al, diclofenac sodium seems to have a higher rate of transport than diclofenac DEA, which is related to the higher saturation solubility of diclofenac sodium. ${ }^{14}$ Formulation composition such as choice of vehicle (solutions, gels, ${ }^{15}$ emulsions, microemulsions, ${ }^{16,17}$ particles, ${ }^{18}$ liposomes, ${ }^{19,20}$ and transfersomes ${ }^{21}$ ) and inclusion of penetration enhancers and characteristics such as water solubility and acidity may influence the ability of topical formulations to permeate the skin and deeper tissues. ${ }^{10}$ To demonstrate the importance of the formulation parameters and composition regardless of the drug concentration, an in vitro skin permeation study was undertaken to compare the permeability of 2 commercially available topical products: diclofenac diethylamine (DEA) $1.16 \%$ emulsion (GlaxoSmithKline, Munich, Germany), which corresponds to $1 \%$ of diclofenac sodium $(\mathrm{Na})$, and diclofenac Na 5\% gel (Sandoz, Holzkirchen, Germany). The qualitative composition and physical characteristics of diclofenac DEA 1.16\% emulsion and diclofenac Na 5\% gel were also assessed in order to provide further insight, which could explain observed differences in skin permeation.

\section{Materials and methods}

\section{Permeability assessment}

The in vitro study used Franz diffusion cells $\mathrm{s}^{22}$ to compare the permeability of diclofenac DEA $1.16 \%$ emulsion and diclofenac Na 5\% gel through ex vivo human skin obtained from the abdominal region of 5 patients during their plastic surgery. The skin collection was approved by two ethical committees (Lothian Research Ethics Committee, Edinburgh, UK, and West of Scotland Research Ethics Services, Glasgow, UK). Written informed consent was obtained from all donors after explaining the purpose of the collection. After collection, skins were frozen at $-20^{\circ} \mathrm{C}$ until their use for the percutaneous permeation study. The day of the experiment, skins were thawed and dermatomed at $\sim 400 \mu \mathrm{m}$ thickness, starting from the stratum corneum. The barrier integrity of each skin sample was confirmed before application of each formulation using the electrical resistance method, and those skin samples with an electrical resistance of $>10.9 \mathrm{k} \Omega$ were included. A single, fixed, $10 \mathrm{mg} / \mathrm{cm}^{2}$ dose (corresponding to the singleapplication dose for topical products recommended in the patient information leaflets) of each diclofenac formulation was applied to a $0.64 \mathrm{~cm}^{2}$ area of the stratum corneum surface of skin samples, which were maintained at $32 \pm 1^{\circ} \mathrm{C}$ and mounted in static diffusion cells. Each formulation was applied to 15 skin samples (ie, 3 replicates were performed for each donor; 30 samples in total). No formal sample size calculation was performed, and the sample size was chosen for exploratory purposes. Receptor fluid (PBS with 5\% w/v bovine serum albumin) samples were collected at $0,2,4,8$, 16 , and $24 \mathrm{hrs}$ after application and analyzed by liquid chromatography/tandem mass spectrometry to quantify permeation of diclofenac (lower limit of quantification, $1 \mathrm{ng} / \mathrm{mL}$ ).

\section{Data and statistical analysis}

Because skin permeability data have been shown to be lognormally distributed, ${ }^{23}$ log-transformed mean cumulative absorption of diclofenac at $24 \mathrm{hrs}$ was compared post hoc between the 2 formulations using a restricted maximum likelihood estimation-based, mixed-effects model, with formulation as a fixed effect and donor as a random effect. Ninetyfive percent CIs for the geometric mean ratios on the original scale were derived by back-transforming the CIs for the differences between formulations on the log-transformed scale. The percentage of applied dose was calculated as follows: $\mathrm{CA}_{24 \mathrm{~h}} /(\mathrm{Q} \times \mathrm{P} / \mathrm{A})$, where $\mathrm{CA}_{24 \mathrm{~h}}$ is the cumulative absorption at $24 \mathrm{hrs}\left(\mathrm{mg} / \mathrm{cm}^{2}\right), \mathrm{Q}$ is the quantity of topical product applied on the skin sample (mg), $\mathrm{P}$ is the percentage of diclofenac in the topical product applied, and $\mathrm{A}$ is the surface area of the skin sample $\left(\mathrm{cm}^{2}\right)$. Flux of diclofenac was calculated at each timepoint:

$$
F_{t}=\frac{C A_{t}}{t}
$$

\section{Physical characterization}

The physical characteristics of diclofenac DEA 1.16\% emulsion and diclofenac $\mathrm{Na} 5 \%$ gel were assessed via microscopic observation and rheological measurement.

\section{Microscopic observation}

A few microliters of each topical product were inspected visually using a Nikon Ni-U microscope (Nikon Instruments, Inc, Melville, NY, USA). 


\section{Rheological characterization}

Rheological measurements of each product were performed on a rheometer MCR 302 (Anton Paar GmbH, Ostfildern, Germany) at $25^{\circ} \mathrm{C}$ using a cone-plate device (CP60-2, $60 \mathrm{~mm}$ diameter, angle $1.995^{\circ}$, truncation $252 \mu \mathrm{m}$ ). Samples were relaxed for 2 mins before measurement. The flow properties were obtained by recording shear rate $\left(\mathrm{s}^{-1}\right)$ and viscosity values $\eta$ (Pa.s) when shearing the sample at increasing shear rates ranging from $1.10^{-3}$ to $2,800 \mathrm{~s}^{-1}$ (logarithmic ramp) for $280 \mathrm{~s}$.

\section{Results}

\section{Permeability assessment}

Diclofenac DEA 1.16\% emulsion exhibited a statistically significant higher permeation through human skin than diclofenac $\mathrm{Na} 5 \%$ gel (geometric mean of cumulative absorption, 554 vs $361 \mathrm{ng} / \mathrm{cm}^{2}$; ratio of adjusted geometric means, 1.54 [95\% CI, 1.14-2.07]; Table 2). When expressed as a percentage of the applied dose permeating human skin at $24 \mathrm{hrs}$, a 7 -fold difference was observed between the diclofenac $\mathrm{Na} 5 \%$ gel and the diclofenac DEA $1.16 \%$ emulsion $(0.077 \%$ vs $0.54 \%$, respectively). Figure 1 shows that the flux of diclofenac $\mathrm{Na} 5 \%$ gel was slightly faster than the one of diclofenac DEA $1.16 \%$ emulsion before $8 \mathrm{hrs}$, after the trend reverted up to $24 \mathrm{hrs}$.

\section{Physical characterization}

\section{Microscopic observation}

Diclofenac Na 5\% gel appeared as a monophasic gel without droplets, whereas diclofenac DEA 1.16\% emulsion

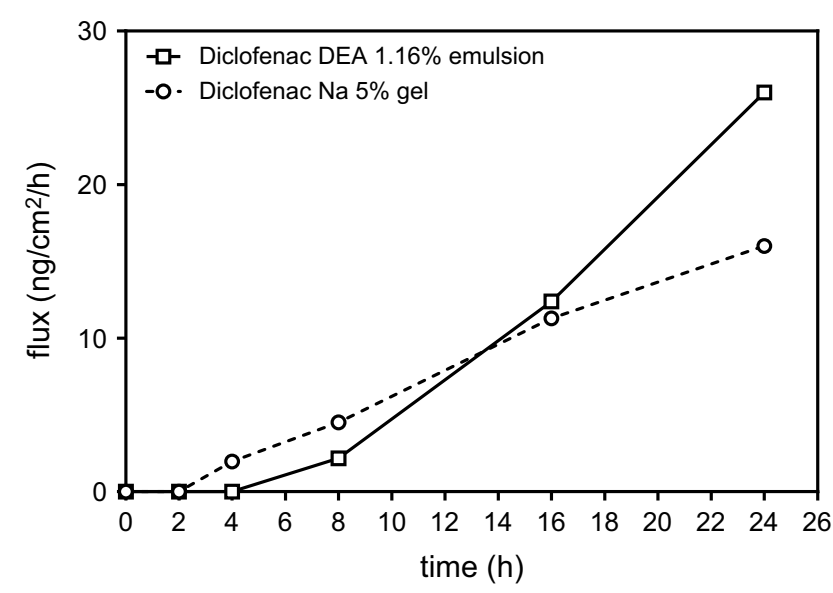

Figure I Median flux of diclofenac over $24 \mathrm{hrs}$.

Abbreviations: $\mathrm{Cl}$, confidence interval; $\mathrm{DEA}$, diethylamine; $\mathrm{Na}$, sodium. demonstrated oily droplets in an aqueous phase, with a narrow distribution of droplet size (mainly $<10 \mu \mathrm{m}$ ).

\section{Rheological characterization}

The viscosity of both products was obtained by applying a controlled shear rate from $1.10^{-3}$ to $2,800 \mathrm{~s}^{-1}$. At 10 $\mathrm{s}^{-1}$, diclofenac Na $5 \%$ gel had a higher viscosity $(24.82$ Pa.s) than diclofenac DEA 1.16\% emulsion (10.29 Pa.s). At higher shear rates, this trend was confirmed.

\section{Discussion}

This in vitro study of human skin permeation of diclofenac, which mimicked the application of a single topical dose typically employed during clinical use, demonstrated significantly greater skin permeation with diclofenac DEA $1.16 \%$ emulsion compared with the higher concentration product, diclofenac Na 5\% gel at $24 \mathrm{~h}$. These results suggest that absorption of topical diclofenac may be influenced by parameters other than drug concentration, and thus, contrary to what might be expected, a higher concentration may not always lead to greater absorption through the skin.

Human skin permeation is likely influenced by the drug physicochemical characteristics (eg, drug salt, drug molecular weight, ${ }^{24,25}$ and $\mathrm{pKa}^{26,27}$ ), the composition of the products (eg, excipients), and the pharmaceutical dosage form (eg, gel, emulsion). The two products contain different salts (diclofenac DEA and diclofenac Na). Therefore, differences in the physicochemical properties, solubility, dissolution rate, and membrane transport between salts can be expected. Despite diclofenac sodium having a higher rate of transport than diclofenac DEA, which is related to the higher saturation solubility of diclofenac sodium, ${ }^{14}$ the data generated showed the opposite. It seems that other parameters could likely be responsible for those unexpected results.

The excipients in topical NSAID formulations likely affect the drug solubility, ${ }^{28}$ diffusion into the formulation, ${ }^{29}$ release from the formulation, ${ }^{30}$ penetration into the stratum corneum, ${ }^{31,32}$ and permeation through deeper skin layers, ${ }^{33}$ thereby influencing its skin absorption. For example, organic solvents, such as isopropyl alcohol and ethanol are known to increase drug solubility and drug release from the formulation. ${ }^{32,33}$ However, the solvent could not be the only influential parameter as it is contained in both formulations and the concentration of the excipients is unknown. The use of permeation enhancers such as propylene glycol (found in diclofenac DEA 
Table I Qualitative composition of diclofenac DEA I.16\% emulsion and diclofenac Na 5\% gel

\begin{tabular}{|l|l|l|}
\hline & $\begin{array}{l}\text { Diclofenac DEA } \\
\text { I.I6\% emulsion }\end{array}$ & $\begin{array}{l}\text { Diclofenac Na } \\
\mathbf{5} \% \text { gel }\end{array}$ \\
\hline Gelling agent & Carbomer & Hypromellose \\
Emulsifier & Macrogol cetostearylic ether & Macrogol glyceryl cocoate \\
Emollient(s) & Liquid paraffin, cocoyl caprilocaprate & Macrogol glyceryl cocoate \\
Permeation enhancer & Propylene glycol & - \\
$\mathrm{pH}$ adjusting & DEA & - \\
Solvents/co-solvents & Purified water, isopropyl alcohol & Purified water, isopropyl alcohol \\
Fragrance & Perfume cream 45 & - \\
\hline
\end{tabular}

Abbreviations: DEA, diethylamine; $\mathrm{Na}$, sodium.

Table 2 Adjusted* geometric mean cumulative absorption at 24 hrs by formulation

\begin{tabular}{|l|l|l|}
\hline Formulation & $\begin{array}{l}\text { Geometric mean } \\
\mathbf{( 9 5 \% ~ C l ) , ~} \mathbf{n g} / \mathbf{c m}^{\mathbf{2}}\end{array}$ & $\begin{array}{l}\text { Diclofenac DEA I.16\% emulsion vs diclofenac Na 5\% gel: } \\
\text { ratio of geometric means (95\% Cl) }\end{array}$ \\
\hline $\begin{array}{l}\text { Diclofenac DEA 1.16\% emulsion } \\
\text { Diclofenac Na 5\% gel }\end{array}$ & $\begin{array}{l}554(265-1,158) \\
36 I(172-754)\end{array}$ & $1.54(1.14-2.07)^{* *}$ \\
\hline
\end{tabular}

Notes: *From mixed model analysis with formulation as a fixed effect and donor as a random effect. **Two-sided $P$-value $=0.0067$ for the superiority test of the null hypothesis that the geometric mean ratio is I.

Abbreviations: DEA, diethylamine; $\mathrm{Na}$, sodium.

$1.16 \%$ emulsion only; Table 1) in combination with other excipients may result in increased skin permeation. The presence of emollients such as cocoyl caprylocaprate and paraffin, both found in diclofenac DEA $1.16 \%$ emulsion, can also improve the skin level of hydration by occlusion, which favors drug absorption. ${ }^{34}$

Physical characterization of both diclofenac products was conducted in order to gain insight into the observation of greater skin permeation with diclofenac DEA $1.16 \%$ emulsion. Although both products are semi-solids, they exhibit physical differences that may potentially affect drug absorption.

Rheological measurements revealed that the formulations containing different gelling agents (carbomer in diclofenac DEA $1.16 \%$ emulsion and hypromellose in diclofenac $\mathrm{Na}$ $5 \%$ gel) behave differently. Diclofenac Na 5\% gel has a higher viscosity at $10 \mathrm{~s}^{-1}$ compared with diclofenac DEA $1.16 \%$ emulsion. The polymeric network created in gelified formulations, described by some parameters such as polymer molecular weight, polymer concentration, and viscosity, could retain the drug differently and could influence its release. $^{35}$ Interestingly, hypromellose seems to create a denser polymeric network in the formulation, which could limit the release of the drug according to the data generated. Further, the gelling agent and the pharmaceutical dosage form (emulsion versus gel) appear to influence the drug release from the different formulations. ${ }^{29}$

The pharmaceutical dosage form may also influence the drug delivery and kinetics. ${ }^{29,36,37}$ Usually, drug formulated in biphasic formulation (emulsion, cream, and ointment) needs to partition out of the internal phase through the external phase before reaching the skin surface. This observation has been confirmed by Stahl et al who noticed a faster drug release from gel compared to biphasic pharmaceutical form (eg, cream). Our flux data suggested that this would be likely the case. Regarding the drug delivery through the skin at $24 \mathrm{hrs}$, in this study, the emulsion seemed to favor higher drug permeation than gel. One explanation could be the dense polymeric network created by hypromellose in the gel product.

The potential effects of physicochemical properties, physical properties, and product composition (eg, excipients) on drug absorption were discussed above. However, multiple ingredients' interactions and physiological parameters also must be taken into account as they will together influence the drug absorption.

\section{Conclusion}

We observed that a product with a higher concentration of diclofenac does not necessarily lead to greater skin 
absorption relative to a product with lower concentration of the active ingredient but different characteristics.

Although our study was specific to topical diclofenac, the findings on the influence of the formulation could be applicable to other drugs. As such, these data support previous studies suggesting that development of a topical pain relief product should take into consideration parameters beyond drug concentration, such as formulation composition. As discussed, these critical parameters may influence the ability of a given NSAID to efficiently penetrate first through the stratum corneum and subsequently the lower layers of the skin to exert its local action at the level of soft tissues and/or joints.

\section{Acknowledgments}

The authors gratefully acknowledge Charles River Laboratory (Edinburgh, UK), which performed the in vitro study. This study was sponsored by GlaxoSmithKline Consumer Healthcare, Nyon, Switzerland. Medical writing assistance was provided by Peloton Advantage, Parsippany, NJ, and was funded by GlaxoSmithKline Consumer Healthcare.

\section{Disclosure}

All authors are GlaxoSmithKline Consumer Healthcare, Nyon, Switzerland employees. The authors report no further conflicts of interest in this work.

\section{References}

1. Schug SA, Goddard C. Recent advances in the pharmacological management of acute and chronic pain. Ann Palliat Med. 2014;3 (4):263-275. doi:10.3978/j.issn.2224-5820.2014.10.02

2. Gan TJ. Diclofenac: an update on its mechanism of action and safety profile. Curr Med Res Opin. 2010;26(7):1715-1731. doi:10.1185/ 03007995.2010.486301

3. Food and Drug Administration. FDA drug safety communication: FDA strengthens warning that non-aspirin nonsteroidal anti-inflammatory drugs (NSAIDs) can cause heart attacks or strokes. 2015. Available from: http://www.fda.gov/drugs/drugsafety/ ucm451800.htm. Accessed October 10, 2018.

4. FitzGerald GA, Patrono C. The coxibs, selective inhibitors of cyclooxygenase-2. $N$ Engl $J \quad$ Med. 2001;345(6):433-442. doi:10.1056/NEJM200108093450607

5. Bjarnason I, Gastrointestinal safety of NSAIDs and over-the-counter analgesics. Int J Clin Pract Suppl. 2013;178:37-42. doi:10.1111/ ijcp. 12048

6. Laine L. Gastrointestinal effects of NSAIDs and coxibs. J Pain Symptom Manage. 2003;25(2 Suppl):S32-S40.

7. Simon LS, Grierson LM, Naseer Z, Bookman AA, Zev Shainhouse J. Efficacy and safety of topical diclofenac containing dimethyl sulfoxide (DMSO) compared with those of topical placebo, DMSO vehicle and oral diclofenac for knee osteoarthritis. Pain. 2009;143(3):238-245. doi:10.1016/j.pain.2009.03.008
8. Tiso RL, Tong-Ngork S, Fredlund KL. Oral versus topical Ibuprofen for chronic knee pain: a prospective randomized pilot study. Pain Physician. 2010;13(5):457-467.

9. Underwood M, Ashby D, Cross P, et al. Advice to use topical or oral ibuprofen for chronic knee pain in older people: randomised controlled trial and patient preference study. BMJ. 2008;336 (7636):138-142. doi:10.1136/bmj.39399.656331.25

10. Hagen M, Baker M. Skin penetration and tissue permeation after topical administration of diclofenac. Curr Med Res Opin. 2017;33 (9):1623-1634. doi:10.1080/03007995.2017.1352497

11. Deng ZH, Zeng C, Yang Y, et al. Topical diclofenac therapy for osteoarthritis: a meta-analysis of randomized controlled trials. Clin Rheumatol. 2016;35(5):1253-1261. doi:10.1007/s10067-015-3021-z

12. Derry S, Moore RA, Gaskell H, McIntyre M, Wiffen PJ. Topical NSAIDs for acute musculoskeletal pain in adults. Cochrane Database Syst Rev. 2015;6:CD007402.

13. Derry S, Conaghan P, Da Silva JA, Wiffen PJ, Moore RA. Topical NSAIDs for chronic musculoskeletal pain in adults. Cochrane Database Syst Rev. 2016;4:CD007400.

14. O'Connor KM, Corrigan OI. Comparison of the physicochemical properties of the $\mathrm{N}$-(2-hydroxyethyl) pyrrolidine, diethylamine and sodium salt forms of diclofenac. Int J Pharm. 2001;222(2):281-293.

15. Seht BL. Comparative pharmacokinetics and bioavailability study of percutaneous absorption of diclofenac from two tropical formulations containing drug as a solution gel or as an emulsion gel. Arzneimittelforschung. 1992;42(2):120-122.

16. Kweon JH, Chi SC, Park ES. Transdermal delivery of diclofenac using microemulsions. Arch Pharm Res. 2004;27(3):351-356.

17. Thakkar PJ, Madan P, Lin S. Transdermal delivery of diclofenac using water-in-oil microemulsion: formulation and mechanistic approach of drug skin permeation. Pharm Dev Technol. 2014;19 (3):373-384. doi:10.3109/10837450.2013.788658

18. Sengupta S, Banerjee S, Sinha B, Mukherjee B. Improved skin penetration using in situ nanoparticulate diclofenac diethylamine in hydrogel systems: in vitro and in vivo studies. AAPS PharmSciTech. 2015;17(2):307-317. doi:10.1208/s12249-015-0347-4

19. Kriwet K, Müller-Goymann CC. Diclofenac release from phospholipid drug systems and permeation through excised human stratum corneum. Int J Pharm. 1995;125(2):231-242. doi:10.1016/03785173(95)00130-B

20. Manconi M, Caddeo C, Sinico C, et al. Penetration enhancer-containing vesicles: composition dependence of structural features and skin penetration ability. Eur J Pharm Biopharm. 2012;82 (2):352-359. doi:10.1016/j.ejpb.2012.06.015

21. Cevc G, Blume G. New, highly efficient formulation of diclofenac for the topical, transdermal administration in ultradeformable drug carriers, Transfersomes. Biochim Biophys Acta Biomembr. 2001;1514 (2):191-205. doi:10.1016/S0005-2736(01)00369-8

22. Franz TJ. Percutaneous absorption on the relevance of in vitro data. J Invest Dermatol. 1975;64(3):190-195.

23. Williams AC, Cornwell PA, Barry BW. On the non-Gaussian distribution of human skin permeabilities. Int J Pharm. 1992;86(1):69-77. doi:10.1016/0378-5173(92)90032-W

24. Brown MB, Martin GP, Jones SA, Akomeah FK. Dermal and transdermal drug delivery systems: current and future prospects. Drug Deliv. 2006;13(3):175-187. doi:10.1080/ 10717540500455975

25. Brown TN, Armitage JM, Egeghy P, Kircanski I, Arnot JA. Dermal permeation data and models for the prioritization and screening-level exposure assessment of organic chemicals. Environ Int. 2016;94:424-435. doi:10.1016/j.envint.2016.05.025

26. Hillery A, Park K. Drug Delivery: Fundamentals and Applications. 2nd ed. Boca Raton, FL, USA; CRC Press: 2017.

27. Jain N. Controlled and Novel Drug Delivery. 1st ed. New Delhi (India): CBS Publishers and Distributors; 2017. 
28. Lee PJ, Ahmad N, Langer R, Mitragotri S, Prasad Shastri V. Evaluation of chemical enhancers in the transdermal delivery of lidocaine. Int J Pharm. 2006;308(1-2):33-39. doi:10.1016/j.ijpharm.2005.10.027

29. Stahl J, Wohlert M, Kietzmann M. The effect of formulation vehicles on the in vitro percutaneous permeation of ibuprofen. $B M C$ Pharmacol. 2011;11:12. doi:10.1186/1471-2210-11-12

30. Flynn GL, Yalkowsky SH, Roseman TJ. Mass transport phenomena and models: theoretical concepts. J Pharm Sci. 1974;63(4):479-510.

31. Florence AT, Tucker IG, Walters KA. Interactions of nonionic polyoxyethylene alkyl and aryl ethers with membranes and other biological systems. In: Structure/Performance Relationships in Surfactants. Vol. 253. Washington, DC, USA: American Chemical Society; 1984:189-207.

32. Benson HA. Transdermal drug delivery: penetration enhancement techniques. Curr Drug Deliv. 2005;2(1):23-33.
33. Loftsson T, Somogyi G, Bodor N. Effect of choline esters and oleic acid on the penetration of acyclovir, estradiol, hydrocortisone, nitroglycerin, retinoic acid and trifluorothymidine across hairless mouse skin in vitro. Acta Pharm Nord. 1989;1(5):279-286.

34. Buck D, Guy R, Maibach H. Effects of occlusion. In: Bronaugh RL, Maibach HI, editors. Vitro Percutaneous Absorption: Principles, Fundamentals and Applications. CRC Press: 1991:85-114.

35. Kandavilli S, Nair V, Panchagnula R. Polymers in transdermal drug delivery systems. Pharm Technol. 2002;26:62-80.

36. Hadgraft J, Whitefield M, Rosher PH. Skin penetration of topical formulations of ibuprofen 5\%: an in vitro comparative study. Skin Pharmacol Appl Skin Physiol. 2003;16(3):137-142. doi:10.1159/000069759

37. Padula C, Nicoli S, Pescina S, Santi P. The influence of formulation and excipients on propranolol skin permeation and retention. Biomed Res Int. 2018;2018:1281673. doi:10.1155/2018/1281673

\section{Publish your work in this journal}

The Journal of Pain Research is an international, peer reviewed, open access, online journal that welcomes laboratory and clinical findings in the fields of pain research and the prevention and management of pain. Original research, reviews, symposium reports, hypothesis formation and commentaries are all considered for publication. The manuscript management system is completely online and includes a very quick and fair peer-review system, which is all easy to use. Visit http:// www.dovepress.com/testimonials.php to read real quotes from published authors. 